\title{
Orthodontic Facemask
}

National Cancer Institute

\section{Source}

National Cancer Institute. Orthodontic Facemask. NCI Thesaurus. Code C82088.

A component of a reverse-pull or protraction orthodontic appliance that distributes force across much of the face. 